\title{
Optical Coherence Tomography for Art Conservation \& Archaeology
}

\author{
Haida Liang ${ }^{* a}$, Borislava Peric ${ }^{\mathrm{a}}$, Michael Hughes ${ }^{\mathrm{b}}$, Adrian Podoleanu ${ }^{\mathrm{b}}$, Marika Spring ${ }^{\mathrm{c}}$, David \\ Saunders ${ }^{\mathrm{d}}$ \\ ${ }^{a}$ School of Science and Technology, Nottingham Trent University, Nottingham NG11 8NS, UK \\ ${ }^{\mathrm{b}}$ School of Physical Sciences, University of Kent, Canterbury, CT2 7NR, UK \\ ${ }^{\mathrm{c}}$ Scientific Department, The National Gallery, London WC2N 5DN, UK \\ ${ }^{\mathrm{d}}$ Department of Conservation, Documentation and Science, The British Museum, \\ London WC1B 3DG, UK
}

\begin{abstract}
Optical coherence tomography (OCT) is a fast scanning Michelson interferometer originally designed for in vivo imaging of the eye. In 2004, our group along with two other groups first reported the application of OCT to art conservation and archaeology. Since that time we have been conducting a project to investigate systematically the potential of OCT as a new tool for non-invasive examinations of a wide range of museum objects and to design an OCT optimised for in situ use in museums. Here we present the latest results from this ongoing project, which include the determination of the optimum spectral windows for OCT imaging of paintings and painted objects executed using traditional techniques, and non-invasive imaging of the subsurface stratigraphy of painted layers at multiple wavelengths. OCT imaging in assisting spectral pigment identification and in measuring refractive indices of paint will also be presented to illustrate the potential of the technique.
\end{abstract}

Keywords: optical coherence tomography, low coherence interferometry, art conservation, paint, pigment, varnish, infrared imaging, infrared reflectography, 3D imaging, refractive index

\section{INTRODUCTION}

Optical coherence tomography (OCT) is a non-invasive and non-contact 3D imaging technique based on the Michelson interferometer using a broadband source with fast 2D or 3D scanning. It was first developed in the early 1990s for the in vivo imaging of the eye and skin tissue in the near infrared spectral range ${ }^{1}$. There are two ways of probing into the depth of the sample: physically moving the reference mirror on a translation stage or fixing the reference mirror but measure the fringes in the spectral domain using a spectrometer and Fourier transform it into the spatial domain. The first type is called a time domain OCT (TD-OCT) and the second type is called a Fourier domain OCT (FD-OCT) ${ }^{2}$. A standard TDOCT collects a series of closely-spaced parallel scans in a plane perpendicular to the surface of the object to give a series of cross section images that can be combined to form a 3D tomogram, and an en face image at a given depth can only be obtained after manipulation of the images with software and not while collecting the scans. An alternative type of TD-OCT collects a series of en face 2D images parallel to the surface of the object at gradually increasing depths, which again are combined to form a 3D image ${ }^{3}$. The depth resolution of an OCT at a given wavelength is given by the spectral bandwidth of the illuminating source. In an OCT, the depth (axial) resolution is decoupled from the transverse (en face) resolution making it possible to achieve high depth resolution at a comfortable distance from the object being examined.

In 2004, the application of optical coherence tomography (OCT) to the examination of paintings, porcelain and ancient jade was first reported ${ }^{4,5,6}$. Since then most of the efforts have been concentrated on new applications of OCT to the examination of paintings ${ }^{7,8,9}$. Apart from the non-invasive examination of the stratigraphy of paint and varnish layers, OCT has also been shown to be the most sensitive technique for revealing preparatory sketches or underdrawings beneath paint layers ${ }^{7}$ owing to its high dynamic range and depth selection capabilities. The high speed of acquisition of OCT has proven to be useful in monitoring the laser cleaning of varnish ${ }^{10}$. OCT has also been shown to have the

*haida.liang@ntu.ac.uk; phone 44115848 3448; fax 44115848 6636;

O3A: Optics for Arts, Architecture, and Archaeology

edited by Costas Fotakis, Luca Pezzati, Renzo Salimbeni

Proc. of SPIE Vol. 6618, 661805, (2007) · 0277-786X/07/\$18 · doi: 10.1117/12.726032

Proc. of SPIE Vol. 6618 661805-1 
potential for monitoring the wetting and drying process of varnish and paint ${ }^{8}$, the deformation of canvas as the result of environmental changes such as humidity ${ }^{11}$ and the solvent cleaning of varnish on paintings ${ }^{12}$. It has also been shown that it is possible to obtain non-invasive measurements of refractive indices of varnish layers using $\mathrm{OCT}^{8}$.

Given the initial success, we have started a systematic study to investigate the potential of OCT as a non-invasive technique for a variety of applications to a wide range of museum objects. In this paper, we report some of the new results from this ongoing project. In Section 2, we present results of a trial run of applying a portable FD-OCT on old master paintings from the National Gallery and fragments of non-accessioned objects from the British Museum in a conservation studio of the National Gallery in London. Section 3 is separated into two parts where the first part gives the results of a systematic study of the spectral transparency of historic artists' paint in the visible and near infrared and the second part discusses non-invasive refractive index measurements of some of these paints using OCT. A discussion of the design specifications of an OCT optimized for in situ use in a museum is given in Section 4. In Section 5, we present a dual wavelength OCT and some preliminary results on test samples and finally in Section 6 we present the potential for pigment identification with OCT and reflectance spectroscopy.

\section{EXAMPLES OF OCT IMAGING OF MUSEUM OBJECTS}

At the last Munich conference on optics for art two years ago, we reported our work on OCT applications on paintings based mainly on samples and test paintings. Here we present a trial run using a FD-OCT for in situ examination of old master paintings in a conservation studio of the National Gallery. The FD-OCT operates at $930 \mathrm{~nm}$ with an axial resolution of $6 \mu \mathrm{m}$, transverse resolution of $9 \mu \mathrm{m}$ and a maximum depth range of $1.6 \mathrm{~mm}$. A number of paintings from the National Gallery and a few fragments of different objects from the British Museum were examined. Here we present example scans of a painting and a fragment of degraded glass to illustrate the different problems encountered in scanning a highly scattering material (e.g. a painting) and a transparent material such as glass.

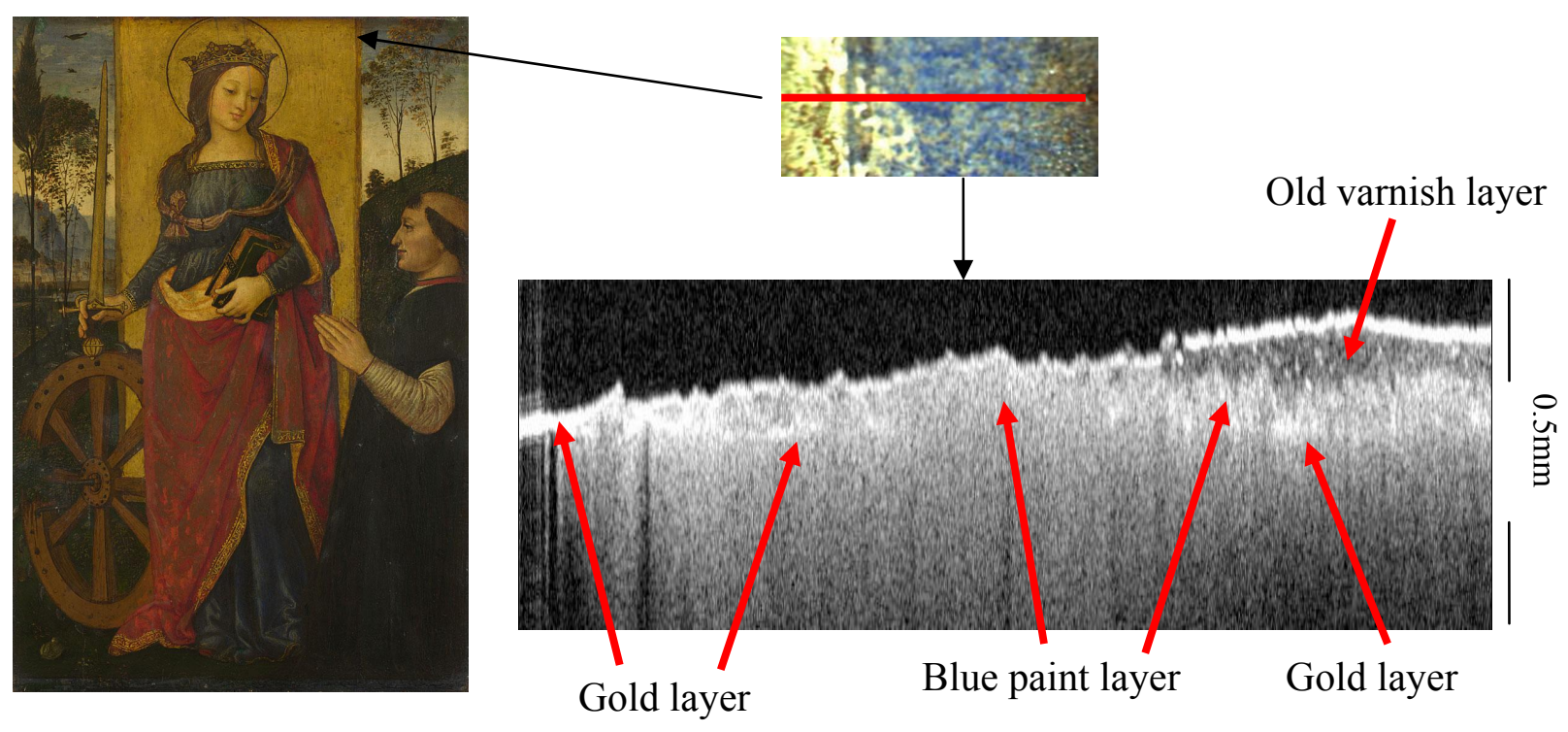

Fig. 1. Left: Saint Catherine of Alexandria with a Donor (1480-1500) by Pintoricchio (National Gallery No. 693); Top right: a detail from the painting where the right hand side is covered with an old yellowed varnish; the old varnish has been cleaned off on the left; the OCT scan direction is marked in red. Bottom right: a 930nm OCT image of a scan through the centre of the area showing the old varnish region on the right and the cleaned area on the left.

Figure 1 shows an example where part of an old degraded yellow varnish has been removed. The painting has an interesting layer structure where paint has been applied over a gold layer. The OCT image shows the varnish, paint and 
gold layers. On the extreme left is the area where the bare gold layer is exposed, in the middle is the blue paint layer (probably ultramarine) over gold and on the right is the area where there is still the old varnish on top of the paint layer. Effects of multiple scattering in the paint layer give the impression of a thick layer below the gold layer. Multiply scattered light has a longer optical path length than singly scattered light from the same depth. Some of the multiply scattered light from the blue paint layer above the gold layer appears to be coming from below the gold layer where the light had only been scattered back once. An example of this effect is shown clearly in Fig. 3 where a layer of madder lake pigment in egg tempera is painted on top of a glass microscope slide. In Fig. 3, both the air/paint and the paint/glass boundaries are clearly discernable, but the image also shows back-scattered light that appears to be from below the paint/glass boundary indicating multiple scattering in the paint layer. This highlights the importance of combining knowledge about the instrument and the painting in interpreting the images. Similarly, caution had to be employed in interpreting cross-section images for biomedical applications. However, paintings are perhaps more varied than the human eye or skin and as the project progresses one of the important outcomes will be the development of experience in interpretation of OCT images from paintings, and a knowledge of which of the various types of stratigraphy or materials encountered in painting are most likely to be successfully imaged by OCT.

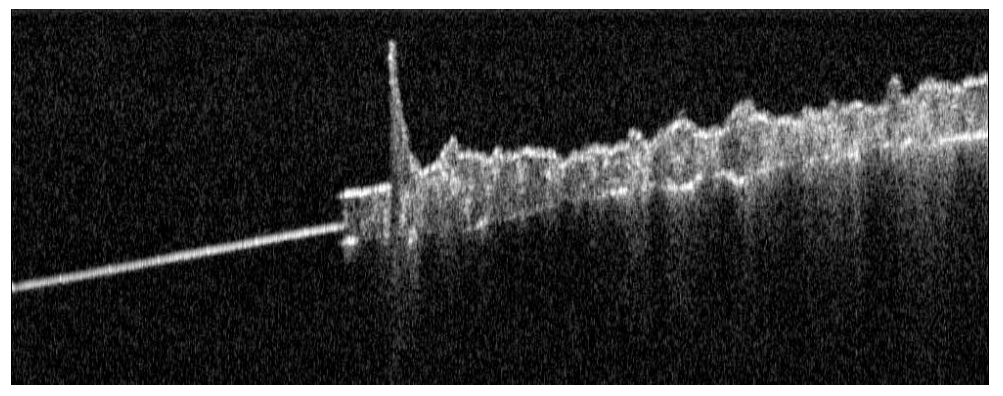

Fig. 3. A 930nm OCT cross section image of madder lake in egg tempera painted on top of a glass microscope slide showing clear evidence for multiple scattering.
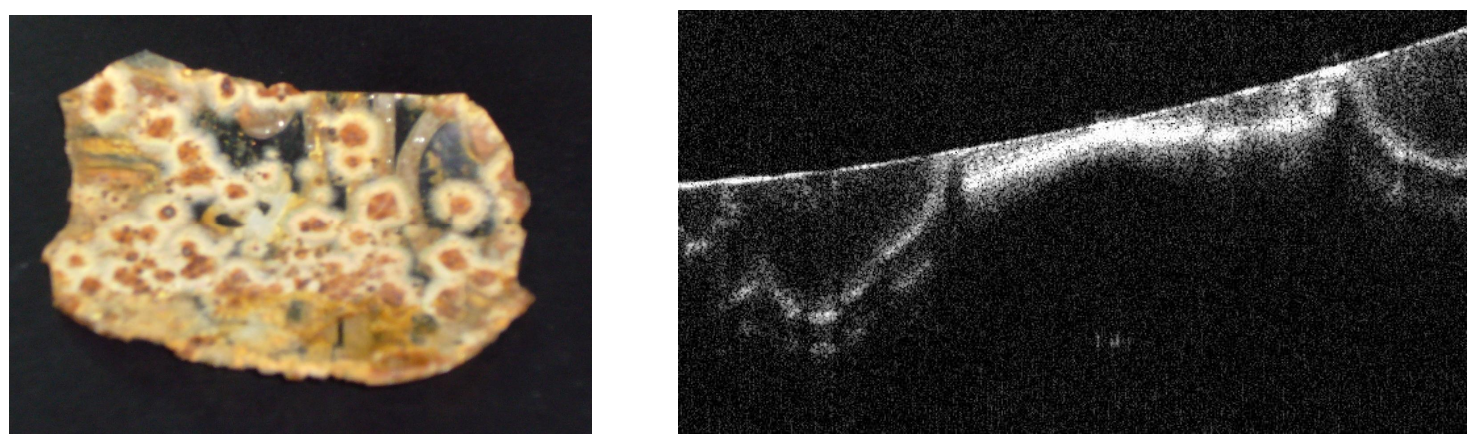

Fig. 4. Left: a piece of $17^{\text {th }}$ Century Antwerp facon de Venise glass showing typical deterioration patterns. Right: an OCT cross-section scan through some of the areas of corrosion.

In contrast, Fig. 4 shows an OCT scan of a highly transparent material, a piece of corroded $17^{\text {th }}$ Century Antwerp facon de Venise glass, a non-accessioned object from the British Museum. The OCT image shows the corrosion spots in cross section where there seem to be multiple corrosion fronts. Note that the glass has a greater optical thickness than the depth range of the OCT, which is why the bottom surface of the glass is not seen in the cross section. One of the limitations of FD-OCT is the short depth range (limited by the number of pixels of spectrometer detector and the depth of focus of the objective lens) ${ }^{13}$ which is usually not a problem for highly scattering material where the scattering limits the penetration depth, but is clearly not adequate for highly transparent material. In the case of the FD-OCT used for this application, the depth range was $1.6 \mathrm{~mm}$ which means it can only image glass up to a maximum thickness of $\sim 1 \mathrm{~mm}$. 


\section{OCT \& OPTICAL PROPERTIES OF PAINT MATERIAL}

\subsection{Spectral Transparency of Traditional Paint}

Since the invention of infrared reflectography for the imaging of underdrawings, there have been studies conducted to directly or indirectly determine the optimum spectral window for infrared imaging ${ }^{14,15,16}$. However, there has not been a comprehensive survey of the transparency of historic artist pigments over the full near infrared (NIR) range. A set of paint-outs consisting of a wide variety of $\sim 50$ historic artists' pigments in both egg tempera and linseed oil has been prepared. The pigments were chosen to be representative of those found on paintings and the compositions of the pigments were verified with EDX, FTIR and XRD measurements. The samples were prepared with known pigment volume concentration and thickness. A list of the pigments is given in Table 1. In order to measure the transparency of the paint layers, one set of paint-outs was prepared over thin glass microscope slides.

Table 1 Pigment List

\begin{tabular}{|c|c|c|c|c|c|}
\hline \multirow{3}{*}{\begin{tabular}{|l} 
Hue \\
red \\
\end{tabular}} & Pigment & Supplier & Hue & Pigment & Supplier \\
\hline & \multirow{2}{*}{$\begin{array}{l}\text { Natural red ochre (French) } \\
\text { Cadmium red } \\
\text { Vermilion } \\
\text { Vermilion light } \\
\text { Sappanwood lake } \\
\text { Lac lake } \\
\text { Red lead } \\
\text { Chrome red } \\
\text { Cochineal lake } \\
\text { Madder lake (from dyed wool) } \\
\text { Madder lake (from ground madder root) } \\
\text { Rose madder (genuine) } \\
\text { Natural iron oxide red }\end{array}$} & \multirow{2}{*}{$\begin{array}{l}\text { Kremer Pigmente } \\
\text { L.Cornelissen and Son } \\
\text { The Pigment Factory Bejing } \\
\text { Kremer Pigmente } \\
\text { prepared by National Gallery } \\
\text { prepared by National Gallery } \\
\text { Kremer Pigmente } \\
\text { Kremer Pigmente } \\
\text { prepared by National Gallery } \\
\text { prepared by National Gallery } \\
\text { prepared by National Gallery } \\
\text { L.Cornelissen and Son } \\
\text { Kremer Pigmente }\end{array}$} & green & $\begin{array}{l}\text { Natural malachite } \\
\text { Artificial malachite } \\
\text { Viridian green } \\
\text { Cobalt turquoise (Rimmans green) } \\
\text { Cobalt bottle green } \\
\text { Verdigris } \\
\text { Bavarian green earth } \\
\text { Phthalo green (Monastral) }\end{array}$ & $\begin{array}{l}\text { Kremer Pigmente } \\
\text { Kremer Pigmente } \\
\text { L.Cornelissen and Son } \\
\text { L.Cornelissen and Son } \\
\text { Kremer Pigmente } \\
\text { Kremer Pigmente } \\
\text { Kremer Pigmente } \\
\text { L.Cornelissen and Son } \\
\end{array}$ \\
\hline & & & \multirow[t]{2}{*}{ blue } & $\begin{array}{l}\text { Smalt } \\
\text { Azurite MP } \\
\text { Azurite } \\
\text { Prussian blue (Milori) } \\
\text { Cerulean blue }\end{array}$ & $\begin{array}{l}\text { L.Cornelissen and Son } \\
\text { Kremer Pigmente } \\
\text { The Pigment Factory Bejing } \\
\text { Kremer Pigmente } \\
\text { Kremer Pigmente }\end{array}$ \\
\hline \multirow[t]{3}{*}{ yellow } & \multirow{3}{*}{$\begin{array}{l}\text { Lemon yellow (barium chromate) } \\
\text { Naples yellow light } \\
\text { Lead tin yellow (type I) } \\
\text { Orpiment } \\
\text { Aureolin (cobalt yellow) } \\
\text { Cadmium yellow deep } \\
\text { Chrome yellow medium } \\
\text { Cadmium yellow light } \\
\text { Dyer's Broom lake } \\
\text { Italian golden ochre } \\
\text { Weld lake } \\
\text { Natural Italian terra di siena (raw) }\end{array}$} & \multirow{3}{*}{\begin{tabular}{|l} 
L.Cornelissen and Son \\
Kremer Pigmente \\
Kremer Pigmente \\
The Pigment Factory Bejing \\
L.Cornelissen and Son \\
L.Cornelissen and Son \\
Kremer Pigmente \\
L.Cornelissen and Son \\
prepared by National Gallery \\
Kremer Pigmente \\
prepared by National Gallery \\
Kremer Pigmente \\
\end{tabular}} & & $\begin{array}{l}\text { Manganese blue } \\
\text { Cobalt blue medium } \\
\text { Artificial ultramarine blue light } \\
\text { Artificial ultramarine blue dark } \\
\text { Indigo }\end{array}$ & $\begin{array}{l}\text { Kremer Pigmente } \\
\text { Kremer Pigmente } \\
\text { Kremer Pigmente } \\
\text { Kremer Pigmente } \\
\text { Kremer Pigmente } \\
\end{array}$ \\
\hline & & & purple & $\begin{array}{l}\text { Manganese violet } \\
\text { Cobalt violet dark } \\
\text { Cobalt violet light }\end{array}$ & $\begin{array}{l}\text { Kremer Pigmente } \\
\text { Kremer Pigmente } \\
\text { Kremer Pigmente }\end{array}$ \\
\hline & & & white & $\begin{array}{l}\text { Titanium white } \\
\text { Lead white }\end{array}$ & $\begin{array}{l}\text { L.Cornelissen and Son } \\
\text { Kremer Pigmente }\end{array}$ \\
\hline
\end{tabular}

The transparency of a paint layer depends on both the scattering and absorption properties, since light is both scattered and absorbed when it travels through the layer. For a strongly scattering paint layer (painted on glass), we expect to find the backscattered light and hence the reflectance to be high and independent of whether the sample was placed on a white or black background. A highly absorbing paint layer would have low reflectance on a white or black background. In contrast, a highly transparent layer will have high reflectance when it is placed on a white background but low reflectance when placed over a black background. For paint layers, the depth penetration of an OCT is limited by multiple scattering rather than absorption since OCT is more sensitive to scattering than absorption.

An Ocean Optics HR2000 fibre optic spectrometer (200-1100nm), a Polychromix DTS 1700 (900-1700nm) and DTS $2500(1700-2500 \mathrm{~nm})$ fibre optic spectrometer were used to measure the spectra between $400 \mathrm{~nm}$ and $2500 \mathrm{~nm}$. The spectral resolutions of the three spectrometers are $0.9 \mathrm{~nm}, 12 \mathrm{~nm}$ and $22 \mathrm{~nm}$. By comparing the spectral reflectance over white and over black, we find that almost without exception all paint samples have highest transparency (or least extinction) at 2.2-2.3 $\mu \mathrm{m}$. There are six pigments that have slightly higher (but comparable) transparency in other regions of the spectra. This is best illustrated by defining a transparency factor $(\eta)$ as

$$
\eta(\lambda)=\frac{S_{W}(\lambda)-S_{B}(\lambda)}{S_{W}(\lambda)}
$$

where $S_{W}$ is the spectral reflectance of the paint measured over a standard white background and $S_{B}$ is the spectral reflectance of the paint when it is placed far away from any reflecting background (this is equivalent to placing it over a non-reflecting black background). Figure 5 shows median transparency of the sample of pigments painted in oil and in egg tempera as a function of wavelength, which illustrates the general increase in transparency into the infrared and that the maximum transparency is at 2.2-2.3 $\mu \mathrm{m}$. Figure 6 shows how the spectral transparency determined from the 
reflectance measurements over white and black background of two paint samples corresponds to their OCT cross section images. The reflectance spectra show that verdigris in linseed oil is highly absorbent at $930 \mathrm{~nm}$ but transparent at $1310 \mathrm{~nm}$ which is confirmed by the OCT images at these two wavelengths. Similarly, the reflectance spectra show that cobalt blue in linseed oil is transparent at $930 \mathrm{~nm}$ but highly absorbent at $1310 \mathrm{~nm}$ which is what we see in the OCT images.

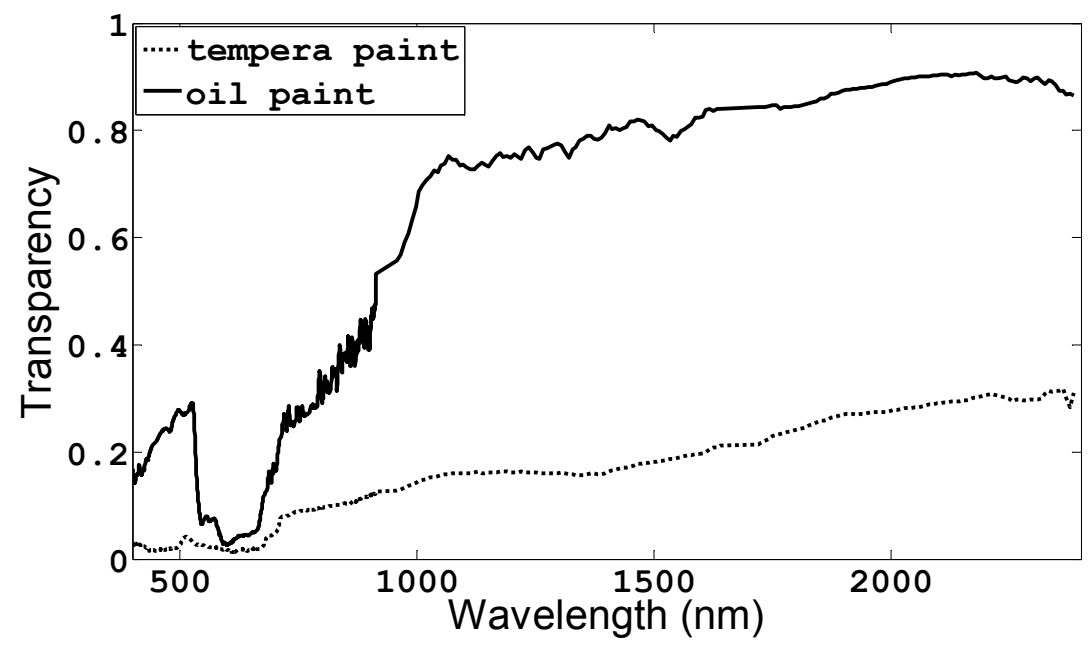

Fig. 5. Transparency $(\eta)$ as defined in Eq. (1) is plotted as a function of wavelength after taking the median transparency of the sample of pigments painted in oil and in egg tempera.

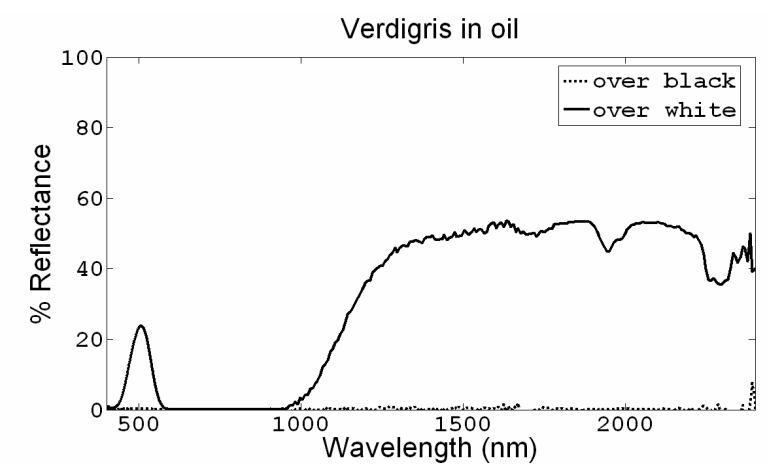

OCT image @1310nm OCT image @ 930nm

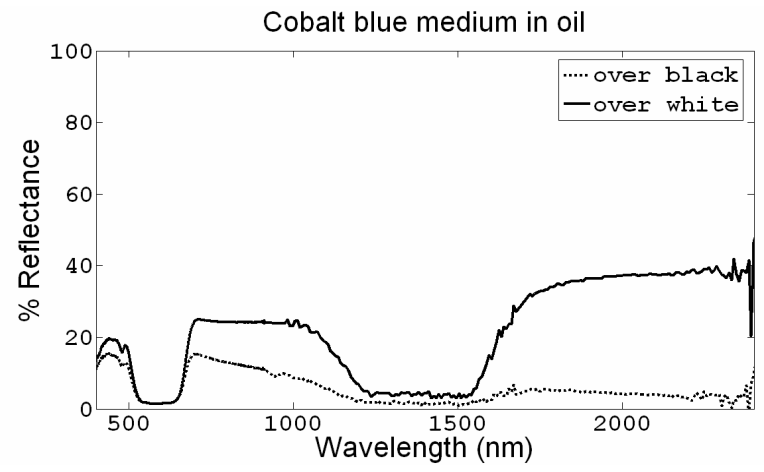

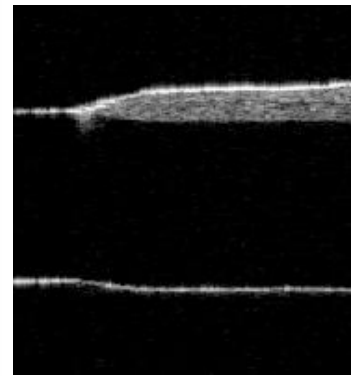

OCT image @ 1310nm

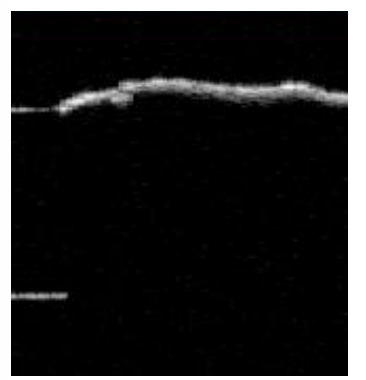

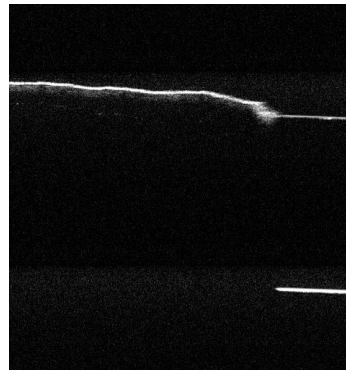

OCT image @ 930nm

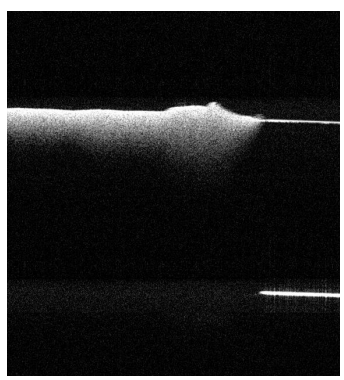

Fig. 6. Spectral reflectance and OCT images at $930 \mathrm{~nm}$ and $1300 \mathrm{~nm}$ of verdigris and cobalt blue medium in linseed oil. The OCT image of verdigris at $1300 \mathrm{~nm}$ is transparent and at $930 \mathrm{~nm}$ it is highly absorbent consistent with the spectral reflectance data. Similarly the OCT image of cobalt blue medium is more absorbent at $1300 \mathrm{~nm}$. 


\subsection{OCT Measurements of Refractive Indices of Traditional Artists' Paint}

Knowledge of the refractive index of paint layers is useful in converting optical thickness to real physical thickness. Refractive indices of the paint samples on glass microscope slides can be measured directly with an OCT. Figure 7 shows one way of measuring the refractive index, where the group refractive index can be measured from the ratio between the optical thickness and the physical thickness of the paint layer as $n=\frac{t_{o}}{t_{r}}$. The measurement is repeated at various points on the paint sample to obtain the mean refractive index and uncertainty. The accuracy of the measurement is limited by the thickness of the paint layer and the depth resolution of the OCT. Another source of error is the overestimation of the optical thickness because of multiple scattering. In order to check if the paint layer is multiply scattering, a second method shown in Fig. 8 measures the optical thickness of the paint layer by subtracting the optical thickness of the glass from the optical thickness of paint and glass and the refractive index is given by

$n=\frac{t_{w}-t_{g}}{t_{r}}$. This second method gives a more accurate measure of the refractive index because it avoids measuring the weak paint/glass interface and measures the well defined air/glass and glass/air interfaces. Similarly, the measurements are repeated at various points on the paint sample to increase accuracy of the measurement. Unfortunately, the second method can not be used with the 930nm FD-OCT because of the limited depth range of the FD-OCT.

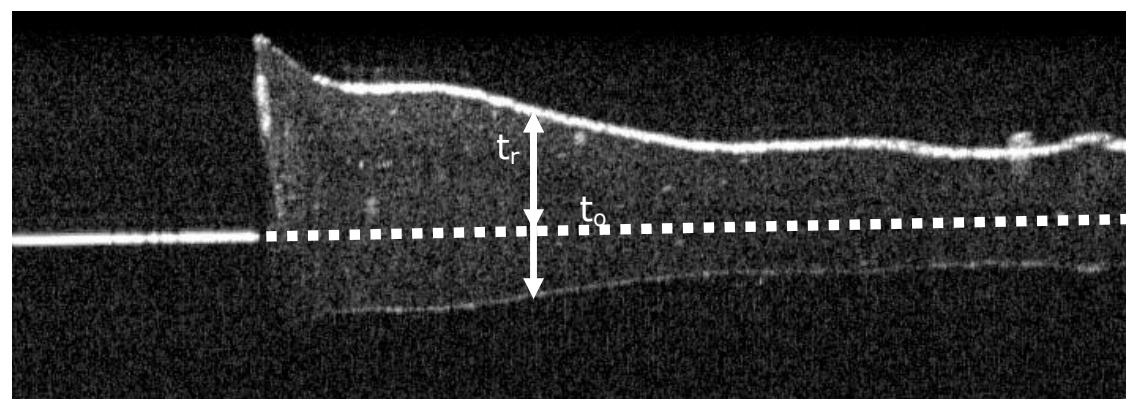

Fig. 7. Refractive index measurement of Rose Madder in linseed oil using a $930 \mathrm{~nm}$ FD-OCT (method 1).

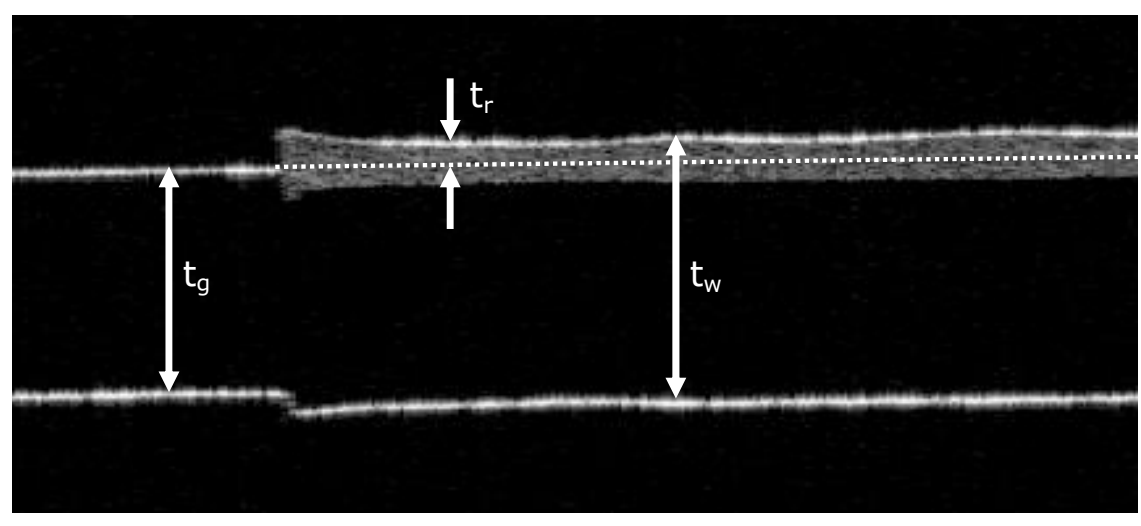

Fig. 8. Refractive index measurement of Rose Madder in linseed oil using a $1310 \mathrm{~nm}$ TD-OCT (method 2).

We present the results of refractive index measurements of two naint samples, cochineal lake in linseed oil and rose madder in linseed oil, both were painted at a wet thickness of $\sim 200 \mu \mathrm{m}$. The measurements were taken with the $930 \mathrm{~nm}$ FD-OCT (depth resolution $6 \mu \mathrm{m}$ ) and a 1310nm TD-OCT (depth resolution $18 \mu \mathrm{m}$ ). For cochineal, the results are 
$n=1.55 \pm 0.02$ at $930 \mathrm{~nm}, n=1.45 \pm 0.12$ at $1310 \mathrm{~nm}$ using the first method and $n=1.53 \pm 0.07$ at $1310 \mathrm{~nm}$ using the second method. Similarly for rose madder, $n=1.59 \pm 0.09$ at $930 \mathrm{~nm}, n=1.59 \pm 0.08$ at $1310 \mathrm{~nm}$ using the first method and $n=1.61 \pm 0.07$ at $1310 \mathrm{~nm}$ using the second method. The uncertainties quoted are one standard deviation. Both paints are in the single scattering regime.

\section{OCT FOR ART CONSERVATION AND ARCHAEOLOGY}

What kind of OCT is best suited to museum applications? In biomedical applications, the demand is for fast OCTs for in vivo imaging which means there is a trade-off between imaging speed and integration time. Current OCTs are mostly designed for biomedical applications where sensitivity is sacrificed for speed ${ }^{13}$. In the case of museum objects, apart from applications such as laser cleaning (Targowski this volume), speed is not crucial as the objects are stationary. We need to re-consider the traditional trade-off in OCT design. In the shot noise limited regime (which is the case for current OCTs), increase of integration time improves the $\mathrm{S} / \mathrm{N}$. It is worth considering either increasing the integration time for OCT acquisition or averaging multiple frames.

Current wisdom considers FD-OCT the future for OCT design because of the higher sensitivity and speed of acquisition. However, for stationary art objects in a studio, speed is no longer a fundamental advantage and FD-OCT has a number of drawbacks compared with TD-OCT. In a FD-OCT, the depth range is limited by the spectral resolution of the spectrometer and the depth of field of the objective lens in the case of high resolution imaging. There is a trade off between the transverse resolution and the depth of field. For example, a transverse resolution of $1 \mu \mathrm{m}$ corresponds to a depth of field of less than $10 \mu \mathrm{m}$ in the near infrared. In the case of TD-OCT, especially en-face OCTs it is possible to overcome the limitation in depth of field by incorporating dynamic focusing mechanism. Another issue is that FD-OCTs are more prone to ghost images, especially when imaging highly reflective surfaces such as freshly varnished paintings. In addition, FD-OCT operating beyond the CCD sensitivity range, i.e. $\lambda>1 \mu \mathrm{m}$ are much more expensive because it needs either an infrared array detector for the spectrometer or a relatively expensive source such as a swept source $\mathrm{OCT}^{17}$.

While museum objects do not move, their environment may not be stationary. In our recent trial run of using a FD-OCT to examine old master paintings in a conservation studio in the National Gallery, we found that vibrations from air conditioning plant as well as soprano voices are noticeable from the OCT scans. Figure 7 (Left) shows the image acquired at the National Gallery of a single cross-section scan consisting of 1000 individual depth scans. The acquisition frequency of the single depth scans is $5 \mathrm{kHz}$ which means one cross-section scan is acquired at 5 frames per second. Figure 7 (Right) shows the average of 11 successive frames which is clearly blurred. Closer examination showed that the frequency of the vibration is less than $\sim 1 \mathrm{kHz}$ (c.f. frequency of human voice is between $\sim 80 \mathrm{~Hz}$ and $\sim 3 \mathrm{kHz}$ ) with a peak-to-peak amplitude of $\sim 20 \mu \mathrm{m}$. For comparison, Fig. 8 shows images acquired by the same OCT in the NTU Imaging Laboratory; the left image is a single cross-section frame and the right image is the average of 11 successive frames which shows no visible blurring. This result shows that vibration effect needs to be taken into account when deciding on the length of the integration time. In situations where vibration can not be avoided, it would be best to average a series of frames of shorter integration time (after some image processing to align the individual frames).
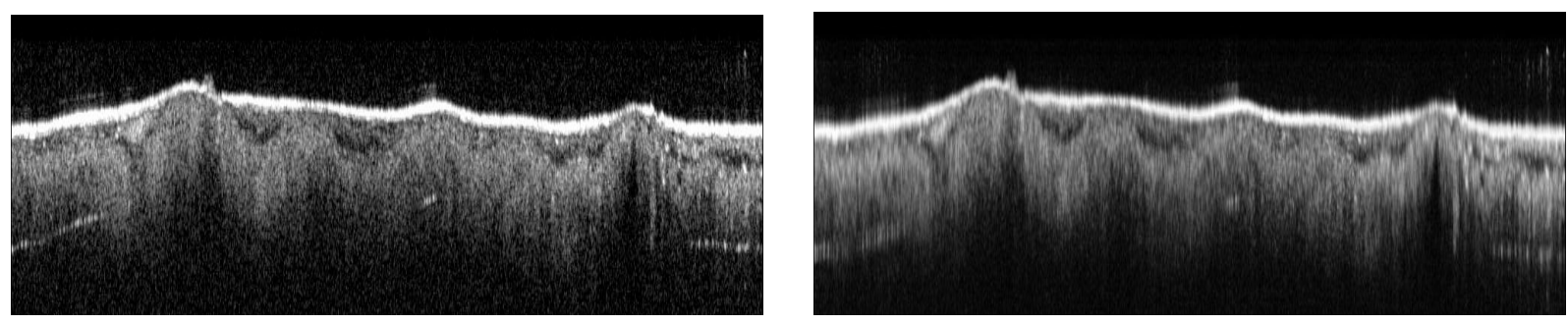

Fig. 7. OCT cross-section images of an area on a large canvas painting in a conservation studio in the National Gallery. Left: one frame Right: average of 11 frames 

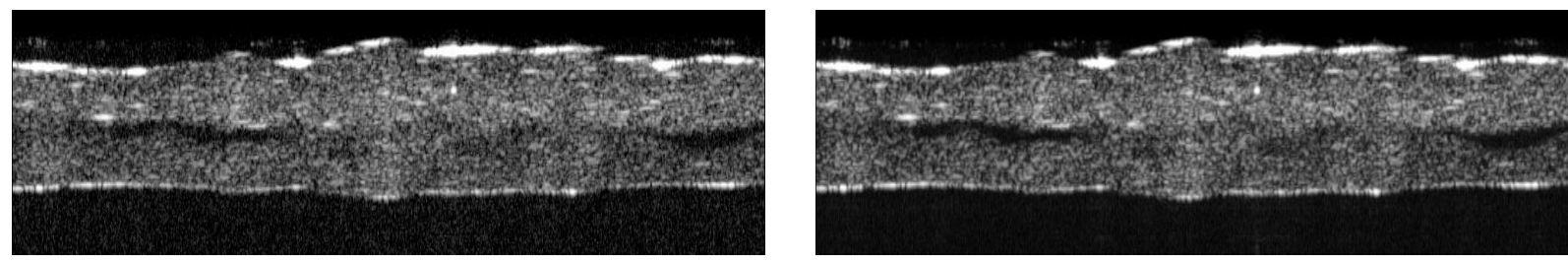

Fig. 8. OCT cross-section images of a paint sample (cochineal lake in linseed oil) on a glass substrate in the NTU Imaging Lab. Left: one frame Right: average of 11 frames

Comparison between images of paint sample cross-section viewed under a microscope and non-invasive subsurface stratigraphy of paintings viewed through an OCT, shows that the limiting factors in information content of an OCT image are the high scattering properties of some of the paint, the relatively low resolution of an average OCT and the lack of colour information. While an average OCT, using an inexpensive SLD source, can give depth resolutions of $\sim 10 \mu \mathrm{m}$ in free space, OCTs using expensive novel broad-band light sources such as Kerr lens mode locked Ti:sapphire laser and photonic crystal fibre based non-linear light sources have been demonstrated to provide $\sim 1 \mu \mathrm{m}$ depth resolution ${ }^{18}$. With such high resolution, it would be possible to resolve many of the pigment particles, making the information on paint structure in the OCT images closer to that from conventional cross sections of paint samples examined with a microscope.

A three-colour wide-field OCT using colour LEDs was recently reported to give colour subsurface images of bees, leaves and apples ${ }^{19}$. Given the high scattering properties of paint in the visible, such a three-colour OCT would have very limited depth penetration in paint. As paint material is more transparent in the near infrared than the visible, a three infrared channel OCT providing false colour cross-section images would be more useful.

While it has been established for a long time that paint material is more transparent in the near infrared than in the visible, it was not clear in which spectral window paint materials are most transparent. The systematic study of the spectral transparency in the visible and the near infrared $(400 \mathrm{~nm}-2400 \mathrm{~nm})$ of paint made of historic artists' pigments and media described in Section 3.1 showed that the best wavelength for transparency to be $\sim 2.2 \mu \mathrm{m}$. An OCT at $\sim 2.2$ $\mu \mathrm{m}$ would be able to probe deeper into traditional paint material than the ones currently available.

\section{A DUAL WAVELENGTH OCT}

A dual wavelength en-face TD-OCT was constructed to test the idea of using multiple wavelength OCT to probe the subsurface structure of paint layers and identify the material. An en-face OCT takes images in planes parallel to the painting surface one after another at increasing depth ${ }^{3}$ which is particularly convenient for the examination of paintings ${ }^{7}$. The OCT is fitted with two SLD sources at $670 \mathrm{~nm}$ and $1300 \mathrm{~nm}$. The setup shown in Fig. 9 enables the acquisition of cross-sectional or en-face scans at both $670 \mathrm{~nm}$ and $1300 \mathrm{~nm}$ simultaneously. The configuration is comprised of two essentially complete OCT systems, whose object arms are superimposed immediately after decoupling from the fibre and prior to scanning by the galvo mirrors. This is achieved using a dichroic beamsplitter, a cold mirror selected with a transition wavelength of $1 \mu \mathrm{m}$. After reflection from the object, the same beamsplitter is used to direct the two wavelengths back into their respective fibre sub-systems. The two systems are further linked by the use of a single translation stage to provide scanning in both reference arms, ensuring that the two channels are synchronised in depth. By placing a mirror in the object arm, the reference optical path for both wavelengths are aligned to within the axial resolution of the system, and hence images obtained for the two channels represent a single spatial region. The axial resolutions are $\sim 20 \mu \mathrm{m}$ in both wavelengths. The transverse resolutions are $10 \mu \mathrm{m}$ and $20 \mu \mathrm{m}$ at $670 \mathrm{~nm}$ and 1300 $\mathrm{nm}$ respectively. 


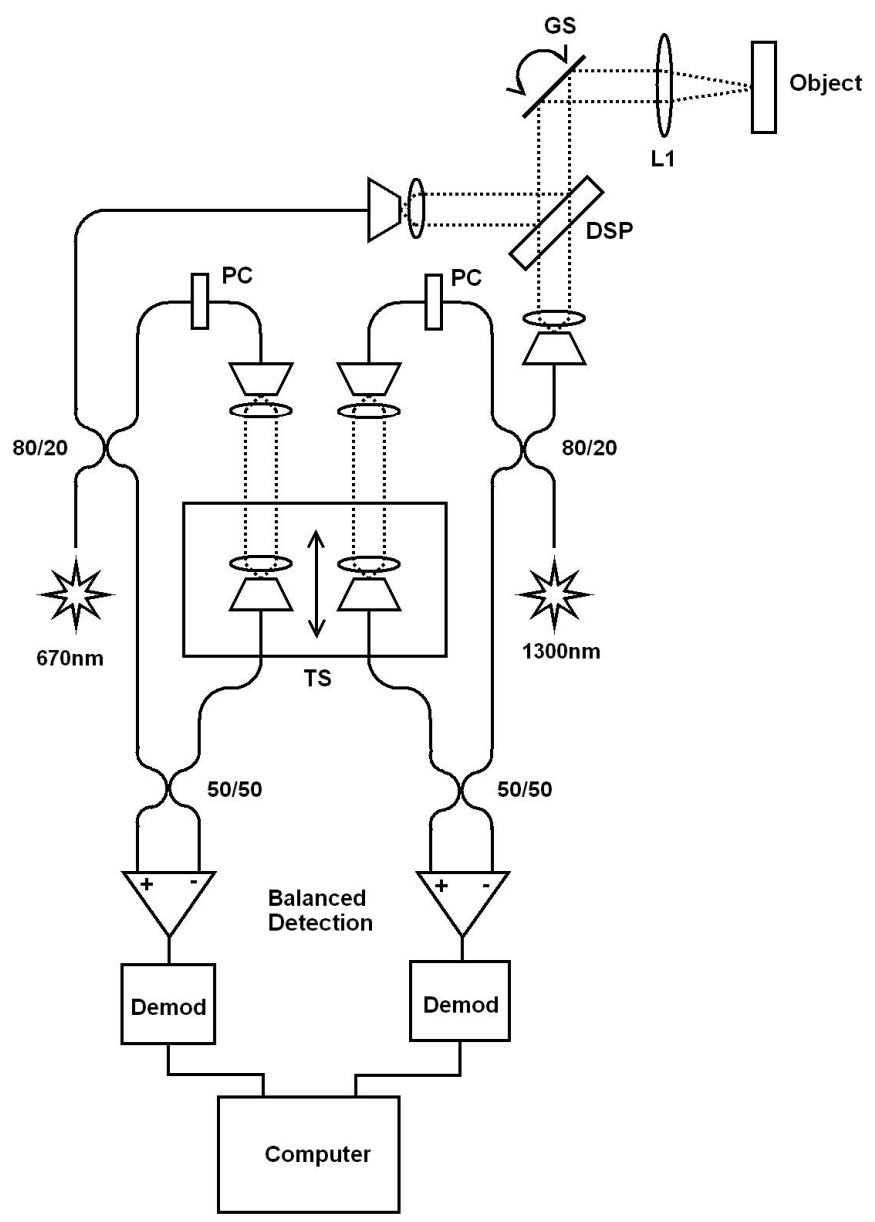

DSP: Dichromatic Beam Splitter

PC: Polaristaion Controller

TS: Translation Stage

Demod: Demodulation Electronics

GS: Galvo Scanners

L1: Object Lens, achromatic doublet

Fig. 9. The dual wavelength optical coherence tomography system architecture

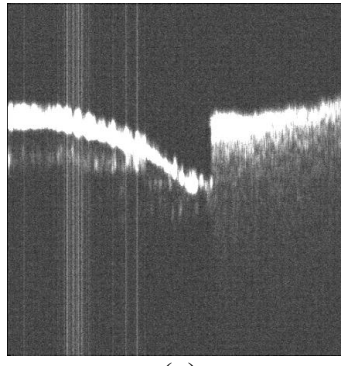

(a)

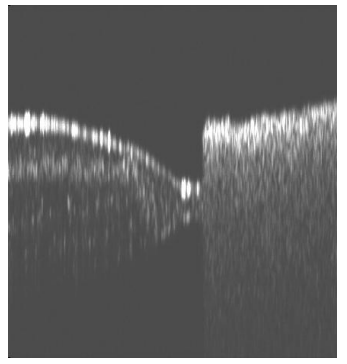

(b)

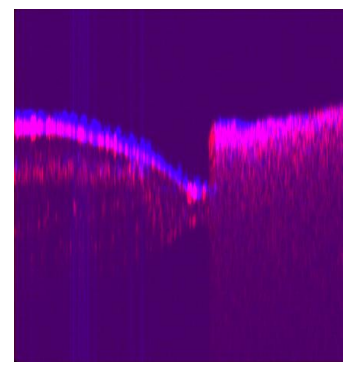

(c)

Fig. 10. Dual wavelength cross section scan of a test sample of Malachite in egg tempera painted over a piece of microscope glass and a piece of white paper as a reference sample. (a) A $670 \mathrm{~nm}$ scan of the test sample (on the left) showing a couple of top layers and a piece of white paper on the right; (b) simultaneous $1300 \mathrm{~nm}$ scan of the test sample and white paper showing 5 layers of the paint; (c) an overlay of the two images where the $670 \mathrm{~nm}$ image is in blue and the $1300 \mathrm{~nm}$ is in red. 
A test sample of malachite in egg tempera (a medium-rich mixture) that was painted in five layers was imaged with the dual wavelength OCT. Figure 10a,b show the simultaneous cross section images at the two wavelengths of the same position on the sample. The $1300 \mathrm{~nm}$ image shows all five layers, but the $670 \mathrm{~nm}$ image shows only the top three layers. The relative intensity of the two images were adjusted such that the white paper used as a reference would give roughly equal backscattered light in the upper portion of the paper where scattering is seen at both wavelengths. The two images are superimposed in Fig. 10c showing the potential for a multiple wavelength OCT scan providing structure as well as "colour" information.

\section{PIGMENT IDENTIFICATION WITH SPECTRAL REFLECTANCE AND OCT DATA}

An alternative method of obtaining both structure and spectral/colour information non-invasively is to combine the structural information from an OCT image with the spectral reflectance measurements (another non-invasive method). Pigment identification using reflectance spectroscopy is both an established technique and one that has not been successful enough to have been met with much enthusiasm in the field of art conservation. The problems are twofold. Firstly, the focus has mainly been on identifying the reflectance spectra of pigments in the visible wavelength region. As such, unique identification of a spectrum to a particular pigment is often difficult as the spectra of pigments of similar hues can look alike. Secondly, it has been difficult to identify mixtures of chromatic pigments with confidence from reflectance spectra alone, especially when it is restricted to the visible spectral range. By extending the wavelength range into the near infrared, additional spectral features that are unique to each pigment are often revealed, allowing more conclusive identification. However, non-invasive spectral pigment identification is still a rather challenging task when it comes to mixtures of pigments. OCT provides additional structural information when the paint layers are translucent and even when they are not, it gives information on the scattering and absorption properties of the material. Similarly, even though for most OCTs, it is currently not possible to resolve the individual pigment particles for most pigments, bulk optical properties of the material can still be extracted. Figure 11 shows an example of OCT images being used to distinguish between a paint of pure smalt in egg tempera and one where it is mixed with a white pigment. The addition of a white pigment does not change the spectral features of a paint mixture significantly as shown in Fig. 11. However, the OCT data shows significant difference between the pure smalt paint and the paint mixed with lead white.

With current technology, it appears that the combination of high resolution spectral data combined with OCT data offers a promising new direction for non-invasive pigment identification. While it does not provide as much of the structural information as is given by a conventional microscope image of a cross section of a sample, it offers more spectral information as well as the additional data on absorption and scattering properties.

\section{CONCLUSIONS}

OCT is the first non-invasive imaging technique to date that allows direct in situ imaging of subsurface structure of works of art. However, research and experience is still needed to interpret OCT images before it can be usefully applied for routine examination. While varnish layers are transparent in the near infrared, many of the paint layers are highly scattering limiting the penetration depth. We have conducted a systematic study of the spectral transparency of historic artitsts' paint and identified the spectral region best suited to OCT imaging of paint layers. In the visible and near infrared (400-2400 nm), paint material is most transparent at $\sim 2.2 \mu \mathrm{m}$. In designing OCTs specific for museum use, we need to re-consider the traditional OCT design criteria meant for in vivo biomedical applications. Museum objects are stationary and hence speed is less important than sensitivity.

Since the initial success of applying OCT to works of art, we are now conducting a systematic study on how to exploit it further in obtaining quantitative information such as measuring the optical properties and assisting non-invasive pigment identification. 

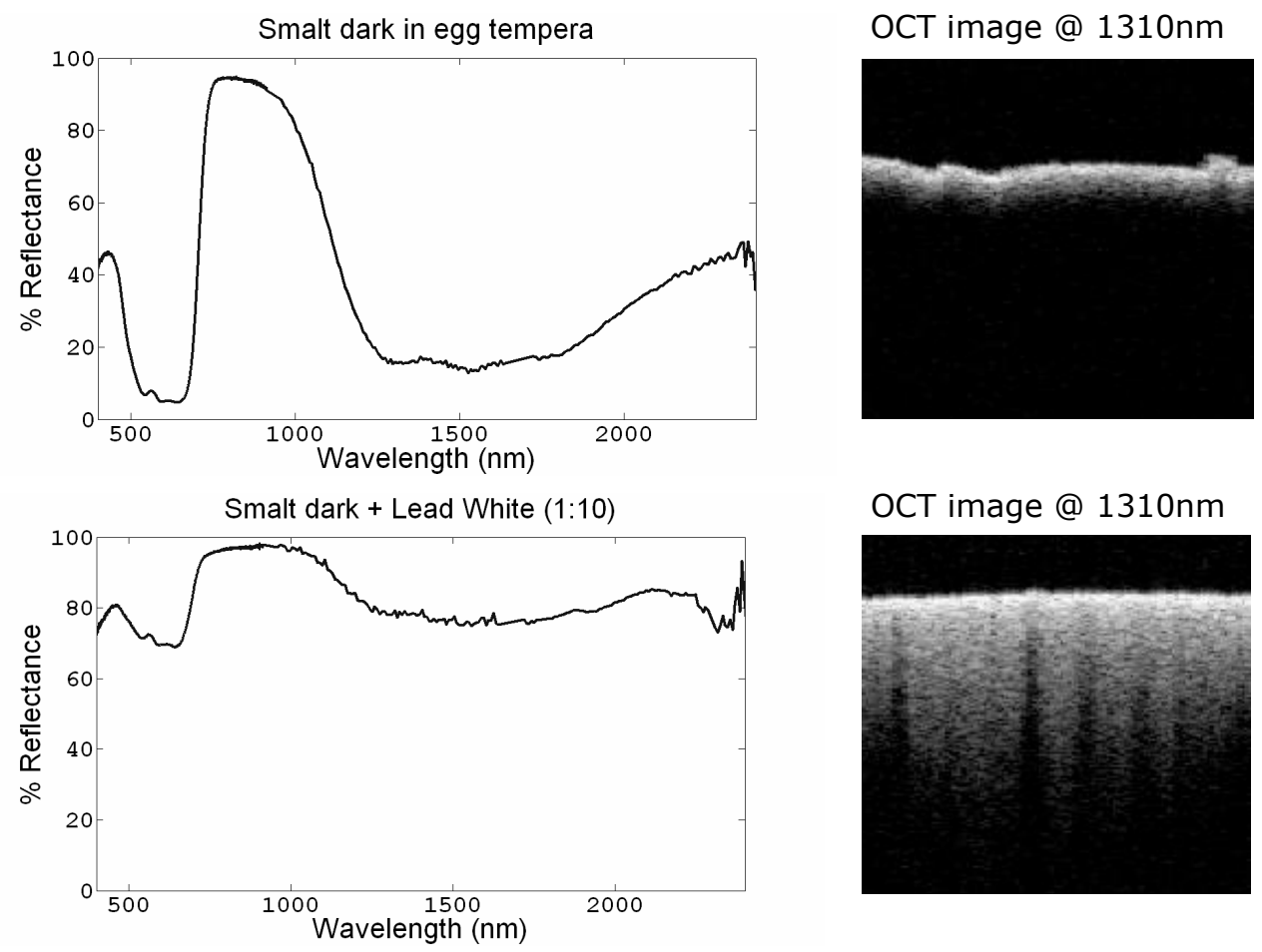

Fig. 11. Top Left: spectral reflectance of pure smalt in egg tempera; Top Right: OCT cross section image at $1300 \mathrm{~nm}$ of the same sample showing low scattering and high absorption. Bottom Left: spectral reflectance of smalt in egg tempera mixed with lead white; Bottom right: OCT cross section image at $1300 \mathrm{~nm}$ of the same sample showing strong scattering because of the presence of lead white;

\section{ACKNOWLEDGEMENT}

This project is funded by the Leverhulme Trust. We would like to thank colleagues at the National Gallery and the British Museum for providing samples, studio space and for useful discussion. We would also like to acknowledge Sophie Martin-Simpson for preparing the historic artists' paint samples.

\section{REFERENCES}

1 D. Huang, E. A. Swanson, C. P. Lin, J. S. Schuman, W. G. Stinson, W. Chang, M. R. Hee, T. Flotte, K. Gregory, C. A. Puliafito, J. G. Fujimoto, "Optical coherence tomography”, Science, 254, 1178, 1991.

2 A. F. Fercher, C. K. Hitzenberger, G. Kamp, S. Y. El-Zaiat, "Measurement of intraocular distances by backscattering spectral interferometry", Opt. Commun. 117, 43, 1995.

3 A. Gh. Podoleanu, J. A. Rogers, D. A. Jackson and S. Dunne, "Three dimensional OCT images from retina and skin", Optics Express, 7(9), 292, 2000.

4 H. Liang, M. Gomez Cid, R. Cucu, G. Dobre, D. Jackson, C. Pannell, J. Pedro, D. Saunders, A. Podoleanu, “Application of OCT to examination of easel paintings", Second European Workshop on Optical Fibre Sensors, Proc. SPIE, 5502, $378,2004$.

5 P. Targowski, B. Rouba, M. Wojtkowski, and A. Kowalczyk, "The application of optical coherence tomography to nondestructive examination of museum objects", Studies in Conservation, 49(2), 107, 2004.

6 M.-L. Yang, C.-W. Lu, I.-J. Hsu, C. C. Yang, "The use of optical coherence tomography for monitoring the subsurface morphologies of archaic jades", Archaeometry, 46(2), 171, 2004. 
7 H. Liang, M. G. Cid, R. G. Cucu, G. M. Dobre, A. Gh. Podoleanu, J. Pedro, D. Saunders, "En-face Optical Coherence Tomography - a novel application of non-invasive imaging to art conservation", Opt. Express, 13, 6133, 2005a. http://www.opticsexpress.org/abstract.cfm?id=85276.

8 H. Liang, M. G. Cid, R. G. Cucu, G. M. Dobre, B. Kudimov, J. Pedro, D. Saunders, J. Cupitt, A. Gh. Podoleanu, "Optical Coherence Tomography - a non-invasive technique applied to conservation of paintings", Proceedings of SPIE, 5857, Optical Methods for Arts and Archaeology, Munich, 2005b

9 P. Targowski, G. Michalina, M. Wojtkowski, "Optical coherence tomography for artwork diagnostics", Laser Chemistry, Article ID 35373, 2006

10 M. Gora, P. Targowski, A. Rycyk, J. Marczak, "Varnish abalation control by optical coherence tomography", Laser Chemistry, Article ID 10647, 2006

11 P. Targowski, M. Gora, T. Bajraszewski et al., "Optical coherence tomography for tracking canvas deformation", Laser Chemistry, Article ID 93658, 2006

12 H. Liang, B. Peric, M. Spring, D. Saunders, M. Hughes, A. Gh. Podoleanu, "Non-invasive imaging of subsurface paint layers with optical coherence tomography", Conservation Science 2007, Milan.

13 P. H. Tomlins and R. K. Wang, "Theory, developments and applications of optical coherence tomography”, J. Phys. D: Appl. Phys., 38, 2519, 2005.

14 J. R. J., van Asperen de Boer, "Reflectography of paintings using an infra-red vidicon television system", Studies in Conservation, 14, 96, 1969

15 J. Delaney, C. Metzger, E. Walmsley, C. Fletcher, "Examination of the Visibility of Underdrawing Lines as a Function of Wavelength", in ICOM Committee for Conservation, 10th Triennial Meeting, Washington DC, 15-19, 1993

16 M. Gargano, N. Ludwig, G. Poldi, "A new methodology for comparing IR reflectographic systems", Infrared Physics \& Technology, 49, 249, 2007.

17 R. Huber, M. Wojtkowski, K. Taira, J. G. Fujimoto, “Amplified, frequency swept lasers for frequency domain reflectometry and OCT imaging: design and scaling principles", Opt. Express, 13, 3513, 2005

18 A. Unterhuber, B. Povazay, K. Bizheva et al., "Advances in broad bandwidth light sources for ultrahigh resolution optical coherence tomography”, Phys. Med. Biol., 49, 1235, 2004

19 L. Yu and M. K. Kim, "Full color three-dimensional microscopy by wide-field optical coherence tomography", Opt. Express, 12, 6632, 2004. 